\title{
Philosophiques
}

\section{Nature et esprit chez Hegel : amant ou rival ?}

\section{Éric Guay}

Volume 30, numéro 2, automne 2003

URI : https://id.erudit.org/iderudit/008647ar

DOI : https://doi.org/10.7202/008647ar

Aller au sommaire du numéro

Éditeur(s)

Société de philosophie du Québec

ISSN

0316-2923 (imprimé)

1492-1391 (numérique)

Découvrir la revue

Citer cet article

Guay, É. (2003). Nature et esprit chez Hegel : amant ou rival ? Philosophiques, 30(2), 391-405. https://doi.org/10.7202/008647ar

\section{Résumé de l'article}

La notion d'« Esprit " que chérit le système hégélien a été tellement galvaudée qu'on n'associe souvent de nos jours ce dernier qu'à l'opposé de la nature sans trop comprendre ce que cela signifie vraiment en termes hégéliens. Dans un même élan, on prend un malicieux plaisir à soulever le haut niveau d'abstraction de cette philosophie qui, aux dires de ses détracteurs, délaisse complètement ce qui est naturel au profit de ce qui est spirituel. La nature et le monde matériel se trouvent-ils si mal servis par l'hégélianisme ? Nous allons montrer que non, en expliquant que cette apparence dérive directement d'un refus de prendre en compte la métaphysique qui oeuvre à l'arrière-plan du désir hégélien d'articuler la totalité de ce qui est dans le discours philosophique. d'utilisation que vous pouvez consulter en ligne.

https://apropos.erudit.org/fr/usagers/politique-dutilisation/ 


\title{
Nature et esprit chez Hegel: amant ou rival ${ }^{1}$ ? ÉRIC GUAY \\ Université du Québec à Trois-Rivières guayeric36o@hotmail.com
}

\begin{abstract}
RÉSUMÉ. - La notion d'«Esprit» que chérit le système hégélien a été tellement galvaudée qu'on n'associe souvent de nos jours ce dernier qu'à l'opposé de la nature sans trop comprendre ce que cela signifie vraiment en termes hégéliens. Dans un même élan, on prend un malicieux plaisir à soulever le haut niveau d'abstraction de cette philosophie qui, aux dires de ses détracteurs, délaisse complètement ce qui est naturel au profit de ce qui est spirituel. La nature et le monde matériel se trouvent-ils si mal servis par I'hégélianisme? Nous allons montrer que non, en expliquant que cette apparence dérive directement d'un refus de prendre en compte la métaphysique qui œuvre à l'arrière-plan du désir hégélien d'articuler la totalité de ce qui est dans le discours philosophique.
\end{abstract}

ABSTRACT. - The notion of "Spirit" so championed in the Hegelian system has been so trivialised, that nowadays one tends to link it to the very opposite of nature without really understanding what it truly means in Hegelian terms. Furthermore, critics take a malicious pleasure in emphasizing the high level of abstraction of this philosophy which, still according to them, results in completely neglecting the natural in favor of the spiritual. Are nature and the material world so poorly served by Hegelianism? We will prove that statement wrong by explaining that this criticism of Hegel's philosophy directly derives from a refusal to consider the metaphysics at work behind the Hegelian desire to thoroughly explain the totality of what is in the philosophical discourse.

L'aimé n'est pas opposé à nous, il forme un seul et même être avec nous; nous ne voyons que nous en lui — et pourtant il n'est pas alors nous-même $[\ldots]^{2}$.

N'allez surtout pas croire qu'une erreur grammaticale s'est sournoisement glissée dans le titre de ce texte et que vous auriez plutôt dû y lire "Nature et esprit chez Hegel: amants ou rivaux?". Car, il est fort opportun de le préciser ici, la nature et l'esprit participent, selon le mode de penser proprement hégélien, à l'expression d'une seule et même chose, entendons l'Absolu ${ }^{3}$. Ce dernier, par définition, ne laisse rien subsister au-dehors de

1. L'esquisse de ce texte a fait l'objet d'une communication présentée dans le cadre du colloque de la Société de philosophie du Québec sur La Phénoménologie et la philosophie de l'esprit contemporaine qui a eu lieu à l'Université du Québec à Montréal les 24 et 25 mars 2000.

2. G. W. F. Hegel, Theologische Jugendschriften, Francfort, Minerva GmbH, 1966, p. 377; L'Esprit du christianisme et son destin, trad. de J. Martin, Paris, Vrin, 1988, p. 140. 
lui lorsqu'il s'affirme. Choyé, sans aucun doute, par la langue allemande avec laquelle il a eu le bonheur de forger ses concepts philosophiques, Hegel parle souvent de la pluralité au singulier. C'est en ce sens bien précis qu'il évoque dans ses écrits - ceux de jeunesse comme de maturité - ce qu'il appelle un Naturgeist, c'est-à-dire un "esprit-nature ». Certains affirment que la conception hégélienne de l'esprit est douteuse et qu'elle se présente comme l'a priori d'un système de pensée qui cache une prépondérance secrète de l'universel sur le particulier ${ }^{4}$. Nous montrerons que ceux qui pensent ainsi n'ont pas été suffisamment attentifs à la théorie hégélienne de l'Esprit. Par contre, nous devons admettre que la théorisation de l'Absolu soutenue par la philosophie hégélienne se complique quelque peu lorsqu'elle soulève la primauté de l'un de ces deux éléments par lesquels nous venons tout juste d'en parler - celui-ci s'exprime. En témoigne ce passage de l'Encyclopédie des sciences philosophiques qui manifeste clairement une prééminence de l'Esprit sur la nature: "L'absolu est esprit — c'est là la définition la plus haute de l'absolu5.» La question à laquelle veut répondre cet exposé est la suivante: si, chez Hegel, l'Esprit, absolument parlant, est supérieur à la nature, pouvons-nous dire que l'Absolu se déteste d'avoir eu à s'abaisser à l'élément naturel tant déprécié par cet auteur? Ou bien, pour formuler la question autrement: est-ce que l'Absolu s'aime toujours d'un égal amour dans la pluralité de ses manifestations parfois dégradantes? L'interrogation est justifiée, parce qu'il faut avouer que ce qui n'est que naturel et immédiat est vertement méprisé par Hegel. Le philosophe va même jusqu'à affirmer, dès le début de sa carrière professorale, que «l'[individu] singulier n'est grand et libre que dans la mesure où son mépris pour la nature est grand ${ }^{6}{ }^{*}$. Nous verrons pourtant qu'en terrain philosophique hégélien, l’Absolu ne peut esquiver la nature, que l'Esprit ne peut éluder l'élément naturel et matériel. Nous montrerons que l'Esprit se sert plutôt adroitement de la nature dans sa définition de soi pour mieux laisser transparaître toute la richesse de sa spiritualité. Afin d'y

3. Nous parerons l'«[A]bsolu» d'une majuscule lorsqu'il exprime vraiment la plénitude et d'une minuscule, "[a]bsolu», dans le cas contraire. Nous ferons de même avec le terme "Esprit».

4. Parmi les interprètes de Hegel qui soutiennent de telles choses, il y a eu Karl Marx et, plus près de nous, Jürgen Habermas, qui se fonde sur le point de vue de Marx pour aborder l'hégélianisme. Voir J. Habermas, La Pensée postmétaphysique. Essais philosophiques, trad. de R. Rochlitz, Paris, Armand Colin, 1993, p. 47.

5. Hegel, Enzyklopädie der philosophischen Wissenschaften im Grundrisse: 1827 (ciaprès Enz.), Gesammelte Werke vol. 19, Hambourg, Felix Meiner, 1989, \$ 384, p. 290; Encyclopédie des sciences philosophiques III Philosophie de l'esprit (ci-après Enc. III), trad. de B. Bourgeois, Paris, Vrin, 1988, $\mathbb{S} 384$, p. 179.

6. Hegel, Schriften und Entwürfe (1799-1808) (ci-après Schriften), Gesammelte Werke, vol. 5, Hambourg, Felix Meiner, 1998, p. 371; Le Premier Système. La Philosophie de l'esprit de 1803-1804 (ci-après P.S.), trad. de M. Bienenstock, Paris, PUF, 1999, p. 34. 
arriver, il faut être attentif à la conception du divin que nous divulgue la philosophie hégélienne.

\section{La totalité divine comme foyer des réflexions hégéliennes sur l'Absolu}

Hegel a très tôt été fasciné par la religiosité. Alors qu'il avait à peine dépassé le cap des dix-huit ans, le jeune Allemand soutenait que «la religion est une des affaires les plus importantes de notre vie ${ }^{7} »$. Cette importance de la religion ne disparaîtra à vrai dire jamais de l'univers hégélien, même lorsque, semblant délaisser cette dernière, notre auteur se consacrera entièrement à la philosophie pour atteindre l'Absolu. Inspirons-nous d'un passage de la Phénoménologie de l'Esprit, que nous trouvons particulièrement éloquent au sujet de la relation qu'entretient la religion avec l'Absolu, pour nous orienter sur la route qui nous amènera à conclure que la nature n'est pas extérieure à l'Esprit dans le discours hégélien:

La vie de Dieu et le connaître divin peuvent donc bien se trouver énoncés comme un jeu de l'amour avec soi-même; cette idée sombre dans l'édification et même dans la fadeur quand y manquent le sérieux, la douleur, la patience et [le] travail du négatif ${ }^{8}$.

Beaucoup de choses pleines de sens viennent d'être dites. Prenons le temps de les démêler afin d'atteindre les perles cachées dans cette philosophie qu'on nous présente trop souvent comme fermée comme une huître. Premièrement, laissons-nous prendre par la référence imagée à la «vie de Dieu et [au] connaître divin" (das Leben Gottes und das göttliche Erkennen). Nous devons savoir que Hegel a la conviction que l'Absolu se présente d'abord à la conscience humaine par des formes divines. Il arrive à cette conclusion en constatant que les divinités sont habituellement les premiers éléments qui expriment ce qui englobe tout ce qui existe pour l'homme. Le philosophe allemand va beaucoup plus loin dans son analyse du divin, estimant que l'Absolu ne se révèle pleinement que dans la religion moderne, à savoir sous la forme du Dieu chrétien. Hegel soutient que la représentation de l'Absolu comme Esprit, qui rayonne de mille feux dans le christianisme, est le «concept le plus sublime [...] qui appartient aux temps modernes et à sa religion ${ }^{9}$ ». Selon le penseur, philosophie et religion sont étroitement liées en raison du but de leur recherche, qui, une fois atteint, éclaire la relation de l'esprit humain et de la nature. Lisons Hegel

7. Hegel, Frühe Schriften I, Gesammelte Werke, vol. 1, Hambourg, Felix Meiner, 1989 , p. 83; "Fragment de Tübingen ", trad. de R. Legros, dans R. Legros, Le Jeune Hegel et la naissance de la pensée romantique, Bruxelles, Ousia, 1980, p. 260.

8. Hegel, Phänomenologie des Geistes (ci-après Ph.G.), Philosophische Bibliothek, vol. 414, Hambourg, Felix Meiner, 1988, p. 14-15; Phénoménologie de l'Esprit (ci-après Ph.E.), trad. de J. Labarrière et G. Jarczyk, Paris, Gallimard, 1993, p. 82.

9. Ph.G., p. 18-19; Ph.E., p. 87. 
qui nous parle justement de philosophie et de religion en un sens bien particulier:

Toutes deux ont pour objet la vérité, et cela dans le sens le plus élevé, — dans celui selon lequel Dieu est la vérité et lui seul est la vérité. Ensuite, toutes deux traitent en outre du domaine du fini, de la Nature et de l'esprit humain, de leur relation l'un à l'autre et à Dieu comme à leur vérité (deren Beziehung auf einander und auf Gott, als ihre Wabrheit ${ }^{10}$.

On pourrait être tenté de croire qu'on subordonne ici la philosophie à la religion, mais il n'en est rien. Pour Hegel, seule la philosophie, en ce qu'elle est apparentée chez lui à la science ${ }^{11}$, peut véritablement comprendre le contenu de la religion. Son contenu - Dieu associé à la vérité - la religion ne le pense pas comme l'Absolu; elle ne fait que l'appréhender d'une manière sensible en ne l'accueillant que sous le mode du sentiment. Ces paroles du philosophe sont on ne peut plus claires: «Le contenu de la religion [...] énonce plus tôt dans le temps que la science ce qu'est l'esprit, mais seule celle-ci est son savoir vrai de lui-même ${ }^{12}$." Rendons à César ce qui est à César. Selon Hegel, c'est bien la religion qui donne à penser à la philosophie, elle lui révèle — à sa manière — l'absoluité d'un réel qui exige inéluctablement le détour du sensible pour resplendir absolument. En fait, nous allons constater que Hegel moule sa philosophie sur le mouvement spéculatif qu'il perçoit dans le christianisme, tout en interprétant ce dernier à partir d'un point de vue logique inédit. Pour en prendre pleinement conscience, revenons au passage de la Phénoménologie de l'Esprit que nous avons commencé à éclaircir. Nous savons désormais que Dieu est associé chez Hegel à la vérité et à l'Esprit, et que cette vérité, la religion ne nous la livre, toujours selon lui, qu'en partie. Permettons-nous, encore une fois, de relire l'extrait dont nous nous sommes inspiré pour débuter notre enquête afin d'en extirper maintenant plus de signification:

La vie de Dieu et le connaître divin peuvent donc bien se trouver énoncés comme un jeu de l'amour avec soi-même; cette idée sombre dans l'édification et même dans la fadeur quand y manquent le sérieux, la douleur, la patience et [le] travail du négatif ${ }^{13}$.

On nous dit donc finalement que Dieu, où l'Absolu qui est Esprit et vérité, peut se trouver énoncé comme ein Spielen der Liebe mit sich selbst,

10. Enz., $\mathbb{S} 1$, p. 27; Hegel, Encyclopédie des sciences philosophiques I. La Science de la logique, trad. de B. Bourgeois, Paris, Vrin, 1986, $\$ 1$, p. 163.

11. Hegel associe, depuis la publication de sa Phénoménologie de l'Esprit, la philosophie à la science dans sa diction de l'Absolu: "Contribuer à ce que la philosophie approche la forme de la science - [...] — c'est là ce que je me suis proposé. » Voir Ph.G., p. 6 ; Ph.E., p. 71.

12. Ph.G., p. 526; Ph.E., p. 687 (nous avons modifié la traduction).

13. Ph.G., p. $14-15$; Ph.E., p. 82. 
" un jeu de l'amour avec soi-même». Le Dieu hégélien serait-il narcissique ? C'est ce qu'on est amené à croire à le voir s'exhiber par un jeu où il s'amourache de lui-même. Par conséquent, Hegel semble nous mettre en garde contre une telle interprétation. L'idée de ce jeu, souligne-t-il, en effet, à la fin du passage que nous continuons à déchiffrer, ne mène pas bien loin s'il lui manque le «sérieux» (der Ernst), la «douleur» (der Schmerz), la "patience et le travail du négatif» (die Geduld und Arbeit des Negativen). Ces éléments, exigés pour esquiver la superficialité du jeu par lequel s'exprime le divin aux yeux de Hegel, font tous référence à un aspect essentiel de la philosophie hégélienne lorsqu'il est concrètement question d'Absolu: die Vermittlung, la médiation. Le mot médiation implique logiquement l'articulation de deux termes différents. Or, c'est précisément sur la logique, ou sur le "royaume de la pensée pure ${ }^{14}$ " (das Reich des reinen Gedankens), comme notre auteur aime l'appeler, qu'est fondé l'Absolu hégélien. On aura l'occasion d'expliquer plus tard de quel genre de "pureté » il s'agit au niveau de la pensée concrète qu'articule le philosophe allemand. Ce qu'il convient de saisir avant tout, en ce qui nous concerne, entendons dans la lignée de notre interrogation de départ, c'est la médiation qui intervient logiquement dans ce «jeu» où l'on dit que Dieu s'y «aime lui-même». Pour ce faire, ce n'est pas sur le «jeu» ou sur l' «amour» qu'il nous faut concentrer notre attention, mais bien sur le fait que Hegel affirme que Dieu puisse être «soi-même» (sich Selbst). Selon le penseur, un "moi », c'est-à-dire ce qui peut être "soi-même ", est toujours le résultat d'un processus qui implique un dessaisissement de "soi ", un détour obligé par le "quelque chose d'autre» qui contribue à donner le relief qui sied à toute ipséité. Une page de la Phénoménologie hégélienne consacrée à Dieu porte sans équivoque sur ce sujet:

Pour qu'en fait il [Dieu] soit Soi et esprit, il lui faut, tout ainsi que l'essence éternelle se présente comme le mouvement d'être égale à soi-même dans son être-autre, devenir à soi-même d'abord un autre (zunächst sich selbst ein Anderes werden $)^{15}$.

L'auteur de ces lignes veut nous faire comprendre que, pour être véritablement conscient de lui-même, Dieu a dû se transformer en monde, que l'Esprit a dû se transformer en nature. Le divin, nous dit Hegel dans la même page où figure le passage que nous venons d'aborder, "se différencie de soi comme autre ou comme monde ${ }^{16}$ " (sich als Andres oder als Welt von sich unterscheidet). D'où provient cette exigence de "se faire autre» à

14. Hegel, Wissenschaft der Logik. Erster Band: Die objective Logik (ci-après W.L. I.), Gesammelte Werke, vol. 11, Hambourg, Felix Meiner, 1978, p. 21; Hegel, Science de la logique. Premier Tome, premier livre: L'Être (ci-après S.L. I.), trad. de J. Labarrière et G. Jarczyk, Paris, Aubier Montaigne, 1972, p. 19.

15. Ph.G., p. 503; Ph.E., p. 657.

16. Ibid. 
laquelle Hegel ose soumettre Dieu lui-même? D'où tire-t-elle sa légitimité ? C'est en logicien qu'il nous incombe de répondre à cette question, puisque cet impératif est associé, par Hegel, à la logique absolue contemporaine que ce dernier affirme livrer dans ses œuvres philosophiques.

\section{La découverte de la logique qui assure le plein rayonnement de Dieu}

En bonne logique hégélienne, il n'y a pas d'identité sans différence. Hegel, dans sa Science de la logique précisément, s'amuse aux dépens de ceux qui clament que l'identité et la différence sont deux choses totalement différentes: "Ils ne voient pas qu'en cela déjà ils disent eux-mêmes que l'identité est quelque chose de divers; car ils disent que l'identité est diverse par rapport à la diversité ${ }^{17}$." Si l'identité est quelque chose de "divers", si l'identité est différente de la différence, c'est donc dire sans détour que l'identité est une "différenciation». C'est soutenir d'un même trait que s'il n'y avait que Dieu, il n'y aurait pas Dieu, qu'un Absolu sans relativité est illusoire, que l'identique doit nécessairement s'enrichir de son «être-autre » pour s'exprimer en vérité. Or, depuis bien longtemps déjà, l'homme a appris à se représenter les divinités comme de purs esprits opposés à ce qui est matériel, en justifiant cette position par le fait qu'on ne peut concrètement faire l'expérience d'une quelconque divinité dans la réalité. Cette "représentation" du divin comme un élément radicalement séparé de la matérialité sensible est broyée par la philosophie hégélienne pour en extirper du sens. Hegel veut nous faire voir que ceux qui envisagent le divin ainsi ne le connaissent pas vraiment. Toujours dans sa Logique, il nous explique que notre façon commune de considérer l'identité comme identique à elle-même peut paraître adéquate dans certains cas, mais qu'elle ne peut atteindre sa cible lorsque l'on tente d'exprimer la vérité de la représentation religieuse de l'Absolu à travers elle. Lisons-le:

En effet, si par exemple à la question: qu'est-ce qu'une plante? on répond: une plante est - une plante, la vérité d'une telle proposition se trouve à la fois concédée par toute la société en laquelle elle se trouve éprouvée, et à la fois on dira de façon tout aussi unanime que par là rien n'est dit. Si quelqu'un ouvre la bouche et promet d'indiquer ce qu'est Dieu, savoir Dieu est — Dieu, l'attente se trouve trompée, car elle envisageait une détermination différente (einer verschiedenen Bestimmung); et si cette proposition est vérité absolue, l'on fait bon marché d'une telle phraséologie absolue; rien ne se trouve tenu pour plus ennuyeux et plus assommant qu'un entretien qui ne fait que rabâcher la même chose, qu'un tel discourir qui pourtant doit être vérité ${ }^{18}$.

17. W.L. I., p. 262 ; Hegel, Science de la logique. Premier tome, deuxième livre: La Doctrine de l'essence (ci-après S.L. II.), trad. de J. Labarrière et G. Jarczyk, Paris, Aubier Montaigne, 1976, p. 41.

18. W.L. I., p. 264 ; S.L. II., p. 43-44. 
Ce Dieu qui se doit d'acquérir une "détermination différente de luimême» pour devenir vraiment lui-même, c'est - comme nous l'avons laissé entendre plus tôt - celui de la religion chrétienne, que Hegel pense à partir du mouvement qui anime la connaissance. Pourquoi Hegel penset-il Dieu à partir de la logique de la connaissance, c'est-à-dire en osant poser la question «qu'est-ce que Dieu » ? Simplement parce qu'il est philosophe, et que la philosophie se détermine comme la discipline par excellence qui vise à connaître. La philosophie, on le sait, selon sa signification étymologique, se dévoile précisément comme "amour du savoir». Mais Hegel ne se contente pas d'aimer le savoir, il souhaite porter cet amour à terme. Le philosophe allemand désire amener la philosophie, comme il le dit si bien, "à pouvoir renoncer à son nom d'amour du savoir et à être savoir effectif ${ }^{9}$ ". Or, le savoir ne se réalise que dans l'universalité. Nous ne savons en effet véritablement quelque chose que lorsque nous pouvons universellement approcher "ce que c'est ", qu'au moment où la connaissance peut se voir partagée par tous les hommes en mesure de raisonner. Ainsi, pour reprendre l'exemple de la Science de la logique de Hegel exposé plus haut, nous pouvons concéder qu'on ne se formalise pas le moins du monde du fait qu'une plante, saisie en sa vérité universelle, ne soit pas exclusivement considérée comme une "plante", mais bien comme un "végétal vivant fixé en terre». Si, dans la connaissance universelle, les choses singulières ont le droit, et même le devoir, de s'exprimer par «quelque chose d'autre», si la «plante» doit absolument se dire différemment pour être connue universellement, il doit en être ainsi de la plus haute conception que l'homme a de Dieu lorsqu'on l'envisage philosophiquement. De la sorte, on ne s'étonnera pas que l'idée de la spiritualité totalement immatérielle du divin qui anime toute religion en vienne à se joindre, par nécessité, selon le point de vue du philosophe, à son altérité absolue, c'est-à-dire à se lier à la matérialité qui s'y oppose absolument. S'il en est ainsi, c'est en raison du type de connaissance à laquelle aspire la philosophie. Cette liaison du spirituel et du naturel, qui semble aller de soi lorsqu'on envisage philosophiquement la religion, se montre avec force dans la Phénoménologie de l'Esprit que Hegel publie en 1807. Certains pourraient nous accuser ici d'interpréter, à tort, la Phénoménologie hégélienne à partir du point de vue d'une œuvre qui lui est contemporaine, à savoir celui de la Science de la logique dont les trois tomes ont été édités entre les années 1812 et 1816 . Pourtant, on prendra conscience que le rapprochement entre les deux ouvrages est pleinement justifié en raison des recherches que Hegel a menées à Iéna peu avant la rédaction du livre qui le consacrera philosophe. C'est ce sur quoi nous allons maintenant nous pencher, afin de dissiper les malentendus qui pourraient provenir du «mouvement de la connaissance » que Hegel met au jour dans sa Logique, dont

19. Ph.G., p. 6 ; Ph.E., p. 71. 
nous affirmons la présence dans la Phénoménologie qui lui est antérieure et qui se donne pour tâche d'entreprendre, comme le précise son auteur, «la présentation du savoir qui apparaît ${ }^{20}$ " (die Darstellung des erscheinenden Wissens).

\section{La période hégélienne d'léna et la figuration d'une «logicité métaphysique»}

L'on peut dire que le savoir, scruté par la philosophie hégélienne, culmine dans l'accomplissement d'une métaphysique absolue, c'est-à-dire à même une connaissance rationnelle et transparente du réel où rien n'est mis à l'écart. Dans un livre publié en 1812, alors qu'il habite Nuremberg, Hegel s'étonne du fait que ce qu' "on appelait métaphysique a été pour ainsi dire extirpé radicalement et a disparu de la liste des sciences ${ }^{21} »$. Nous ne devrions pas trop être surpris de ce que les choses aient tourné de la sorte en Allemagne, surtout lorsque l'on sait combien la métaphysique est devenue suspecte après que Kant l'eut abordée dans sa première Critique comme une chimère fondatrice de la réalité qui dépasse les limites de notre savoir théorique. Quoi qu'il en soit, Hegel soutient, un peu plus loin dans le même livre, avoir réussi à mettre au point une logique qui se confond totalement avec la métaphysique. La science logique, y précise-t-il, «constitue la métaphysique proprement dite (die eigentliche Metaphysik) ou la pure philosophie spéculative ${ }^{22}$ ", tout en ajoutant que cette dernière "s'est vue jusqu'à présent encore très négligée ${ }^{23}$ ». Or, cette association de la logique à la métaphysique, à laquelle procède la Science de la logique hégélienne pour manifester adéquatement l'Absolu, n'est pas l'apanage des travaux hégéliens de Nuremberg. On en retrouve l'amorce dans les cours de Logique et métaphysique que notre auteur dispense à l'université d'Iéna avant de publier sa Phénoménologie de l'Esprit. Laissons à A. Stanguennec le soin de nous renseigner à ce sujet:

Alors que, dans les cours de 1801 à 1803, la logique et la métaphysique conservent une hétérogénéité, c'est la profonde homogénéité des deux qui est établie dans la Logique et Métaphysique de 1804-1805. L’homogénéité des deux disciplines s'établit par une présence réciproque de l'une à l'autre. Présence de l'inconditionné métaphysique dans la logique (de l'essence dans la forme), présence continuée et intériorisée de la dialectique logique dans la métaphysique (de la forme dans l'essence) $)^{24}$.

L'union de la logique à la métaphysique, qui tend à s'affirmer dans les cours hégéliens qui traitent de ces deux disciplines philosophiques, tient

20. Ph.G., p. $60 ;$ Ph.E., p. 135.

21. W.L. I, p. 5; S.L. I, p. 1.

22. W.L. I, p. 7; S.L. I, p. 5.

23. Ibid.

24. A. Stanguennec, Hegel. Une philosophie de la raison vivante, Paris, Vrin, 1997, p. 53. 
principalement à la découverte de la motricité qui anime le savoir. Hegel mise beaucoup sur le mouvement qui s'exhibe dans nos connaissances pour servir sa vision systématique. Il le fait, en considérant le savoir comme l'analogon de l'Absolu vivant que l'on peut percevoir dans toute réalité. Lisons notre auteur qui nous explique justement que le savoir se meut tout en effectuant une certaine réconciliation entre l'identique et le différent:

Le connaître est l'universel en tant que totalité, pour autant qu'en lui le contenu total de l'universel se présente développé; il est la réflexion totale qui demeure purement et simplement égale à soi dans son altération [...]. L'universalité du connaître est elle-même cette forme de l'indifférence dans laquelle survient le contenu, autrement dit il n'y a de contenu que posé sous cette forme indifférente ${ }^{25}$.

Nous sommes incontestablement ici en présence de ce qui a permis à Hegel d'élaborer une logique qui englobe tout, à savoir une logique métaphysique, telle qu'affirme nous la présenter le premier tome de l'un de ses maîtres ouvrages. En vertu de la découverte de cette dynamique, l'on comprendra mieux pourquoi Hegel viendra à considérer l'Absolu par un mouvement selon lequel il se présente initialement comme abstrait, se concrétise par la suite matériellement en devenant Nature, puis se retrouve finalement lui-même en apparaissant comme Esprit. Il suffit, pour y voir plus clair, d'associer la figuration de l'Absolu au mouvement conceptuel qui anime notre connaissance, pour autant, bien sûr, que l'objet de notre savoir témoigne d'une prétention à l'universalitée ${ }^{26}$. Ainsi, dans des notes qui ont sans doute servi aux cours de Philosophie de l'esprit que Hegel donne presque en même temps qu'il enseigne la Logique et métaphysique à Iéna, l'on reconnaît l'union de l'Esprit et de la nature en vertu d'un mouvement où l'Absolu se confond avec une conceptualité qui fraie avec l'altération: "L'esprit n'est que la relève (das Aufheben) de son être-autre. Cet autre, qu'il est lui-même, c'est la nature. L'esprit n'est que ce qui, à partir de cet être-autre, se transforme en un égal à soi-même ${ }^{27}$.» Ce mouvement caractéristique de l'Absolu est justement celui qui anime la Science de la logique hégélienne, dont la première partie paraît - nous l'avons mentionné - en

25. Hegel, Jenaer System... [illisible], Gesammelte Werke, vol. 11, Hambourg, Felix Meiner, p. 122; Logique et métaphysique (Iéna 1804-1805), trad. de D. Souche-Dagues et A. Kaan, Paris, Gallimard, 1980, p. 144-145.

26. Comme l'exemple de la plante présenté au début de notre texte. Une plante universalisée ne se présente pas seulement comme "une plante", mais bien comme "un végétal vivant fixé en terre".

27. Schriften, p. 370 ; P.S., p. 33. Les parenthèses sont de nous. Comme vous pouvez le constater, nous traduirons le verbe ausfleben et ses dérivés à la manière de Derrida, soit par le terme "relever ", au sens où l'on peut être à la fois élevé et relevé de ses fonctions, remplacé dans une sorte de promotion par ce qui suit et prend la relève. Voir J. Derrida, «Le puits et la pyramide ", dans J. Hyppolite, Hegel et la pensée moderne, Paris, Presses Universitaires de France, 1970, p. 53. 
1812. Mais, avant de rédiger cette logique aux visées "métaphysiques", Hegel a publié, nous le savons, son illustre Phénoménologie de l'Esprit. De sa première grande œuvre systématique livrée au public, notre auteur soutient que " [ce] qui se prépare en elle est l'élément du savoir ${ }^{28}$ » (was er in ihr sich bereitet, ist das Element des Wissens), et que "cet élément est la Logique ou Philosophie spéculative ${ }^{29}$ ». Dans la mesure où nous savons désormais, d'une part, que la mise en branle d'une "logique absolue » chez Hegel, c'est-à-dire une logique métaphysique, a présidé à la rédaction de sa Phénoménologie de l'Esprit et d'autre part, que l'auteur de cette dernière nous la décrit précisément comme une introduction à cette Logique qu'il semble déjà avoir l'intention de publier à sa suite, rien ne nous empêche plus de nous inspirer des propos formulés dans la Logique hégélienne de la maturité pour montrer en quoi Esprit et nature participent tous deux à l'émergence d'un unique élément dans l'ouvrage hégélien de 1807, à savoir l'émergence de l'Absolu lui-même. C'est ce que nous allons constater en découvrant la manière dont Dieu vient en aide à la conscience selon Hegel. Pour ce faire, nous nous attarderons à déchiffrer quelques extraits de l'ouvrage par lequel la philosophie proprement hégélienne est née.

\section{Le divin à la rescousse de la conscience dans la Phénoménologie de l'Esprit de Hegel}

Dans sa Phénoménologie de l'Esprit, Hegel affirme suivre pas à pas le développement de la conscience humaine qui, à cause des situations qu'elle a traversées au cours de l'histoire, peut maintenant atteindre l'Absolu. Mais, selon lui, chaque conscience singulière doit refaire l'expérience conscientielle historique à son propre compte pour atteindre ce but. Présentée comme «chemin du doute, ou à parler plus proprement, comme chemin du désespoir ${ }^{30}$ ", la route empruntée par la conscience pour se hisser à l'absoluité du réel n'est pas, on l'aura compris, de tout repos. Si le chemin est ardu, c'est parce que la conscience, en se développant, pour reprendre l'expression de Hegel, "perd sa vérité ${ }^{11}$ " (es verliert seine Wabrheit). La conscience, en tant qu'elle est religieuse, est précisément abordée, dans la Phénoménologie de l'Esprit, comme un palliatif à l'une des pertes de vérité qui l'affligent au cours de son développement. En effet, Hegel fait intervenir la formation de la religion absolue à l'intérieur de la conscience, dans son ouvrage de 1807, comme une instance qui vient pallier le désespoir des hommes qui ont déchanté après avoir cru pouvoir atteindre la vérité, en d'autres termes l'Absolu, en concentrant tous leurs efforts dans la structure politique, ou étatique, du monde. C’est dans l'expérience conscientielle de

28. Ph.G., p. 29; Ph.E., p. 98.

29. Ibid.

30. Ph.G., p. 61; Ph.E., p. 136.

31. Ibid. 
la Révolution française que le penseur allemand nous demande de replonger afin de comprendre quelle est la plus haute fonction historique de la religion, expérience troublante dont il nous incombe maintenant d'esquisser la teneur si nous voulons comprendre le véritable enjeu de la religion pour la conscience aux yeux de Hegel.

Lorsqu'un peuple n'est plus en mesure de tolérer les injustices qu'il perçoit dans les actes de celui qui le dirige par la contrainte, il vient un temps où les hommes qui le composent veulent changer les choses sans plus tarder. Ils sont conscients qu'ils doivent faire quelque chose pour améliorer la situation, sans pour autant savoir vraiment ce qu'un tel changement exige. C'est ce qui s'est passé, selon Hegel, avec les révolutionnaires français, qui, enthousiasmés par leur foi en la possibilité d'un monde où la bonté règne, ont tenté de réconcilier la terre avec le monde paradisiaque qu'ils avaient en tête. Notre auteur soutient que ces derniers n'y sont arrivés qu'en principe: "[Les] deux mondes sont réconciliés, et le ciel amené et transplanté sur la terre ${ }^{32}$ ", mais, souligne-t-il un peu plus loin, "[l]'audelà de cette sienne effectivité flotte au-dessus du cadavre de l'autostance disparue de l'être réel ou objet-de-foi seulement comme l'exhalaison d'un gaz insipide, de l'Être suprême vide (des leeren Être suprême) ${ }^{33}$ ". Ce qu'on veut nous faire comprendre par cette dernière assertion, c'est que les révolutionnaires se sont éloignés de la signification plénière de Dieu, ou de l'Être suprême, en fondant simplement leur devoir d'agir sur une compréhension immédiate de la religion chrétienne, à savoir sur le fait que les hommes sont tous frères et égaux. Cette malencontreuse attitude a eu pour effet de simplement faire disparaitre la structure politique, pourtant essentielle pour que la conscience puisse atteindre réellement la vérité qu'elle cherche inlassablement - ce que Hegel nous présente comme l' "autostance disparue de l'être réel» (der verschwundnen Selbstständigkeit des realen Seins), au lieu de transformer cette structure politique pour qu'elle réponde mieux aux attentes des hommes qui se sentent floués par celui qui les dirige. Lecteur attentif de Rousseau, notre auteur sait fort bien que la liberté ne peut s'étendre absolument qu'au moment où elle accepte de se restreindre, que la liberté ne peut persister que sous la contrainte d'un pacte social fictif avec lequel "on gagne l'équivalent de tout ce qu'on perd, et plus de force pour conserver ce qu'on $\mathrm{a}^{34} »$. Hegel statue, en ce sens, qu' «il n'est donc aucune œuvre positive ni [aucun] acte [positif] que puisse produire au jour la liberté universelle (die allgemeine Freiheit); ne lui reste que l'agir négatif; elle n'est que la furie du disparaître (die Furie des Verschwindens) ${ }^{35}$ ». Si la religion chrétienne est bien venue en aide aux

32. Ph.G., p. 385 ; Ph.E., p. 515.

33. Ph.G., p. 387-388; Ph.E., p. 518.

34. Jean-Jacques Rousseau, Du contrat social, Paris, Garnier-Flammarion, 1966, p. 51 .

35. Ph.G., p. 389; Ph.E., p. 520. Les interpolations sont de nous. 
révolutionnaires français, en ce qu'elle leur a inspiré l'idée que tous les hommes sont, aux yeux de Dieu, égaux, elle ne l'a pas fait selon son contenu vrai, à savoir dans l'optique d'une apparition de la logique absolue où les déterminations se lient réellement à leurs contraires.

Voilà pourquoi l'avènement du Dieu chrétien pour la conscience humaine se présente, selon Hegel, comme un moment clef de la révélation de l'Absolu. La Phénoménologie soutient, répétons-le, que le contenu de la religion, c'est-à-dire Dieu, "énonce plus tôt dans le temps que [ne le fait] la science ce qu'est l'esprit, mais seule celle-ci est son savoir vrai de luimême ${ }^{36}$ ". S'il en est ainsi, ce n'est pas simplement parce que l'on dit que Dieu s'est investi dans la création du ciel et de la Terre - on s'emmurerait de la sorte dans ce panthéisme romantique, cette absolutisation immédiate de la nature que le philosophe allemand veut dépasser parce qu'il ne nous permet pas de connaître véritablement l'Absolu -, mais bien parce que Dieu a tout autant su se faire lui-même homme dans la personne du Christ pour resplendir absolument. Avec le christianisme, l'humanité est impliquée, et non simplement la nature, dans la manifestation de l'Absolu. C'est là un gain incontestable pour une pensée allemande qui vise à ne laisser rien choir en dehors d'elle-même, pour une pensée qui se veut absolue. Ce n'est que par le panthéisme antique, doublé de l'idée de la Menschwerdung (devenir-homme) de Dieu qui anime le christianisme, que peut s'exprimer religieusement l'absolu. L'assimilation de ce double legs religieux démontre que l'Absolu a, pour le religieux, déjà habité tout ce qui est. "Déjà " habité - prenons-nous la peine de souligner - parce que pour le chrétien fondamentaliste, comme le précise Hegel, l'Absolu a malheureusement quitté notre monde, pour lui, «Dieu lui-même est $\operatorname{mort}^{37}$ ». Tout se passe comme si les adeptes du christianisme orthodoxe, trop captivés par le messager divin en tant que tel, avaient manqué la teneur du message livré par ce dernier, à savoir que l'Absolu se présente par un "mouvement selon lequel ce qui est absolument opposé se connaît comme la même-chose $e^{38}$ " (die Bewegung, daß das absolute Entgegengesetzte sich als dasselbe erkennt). C'est ici que la philosophie prend la relève (aufhebt) de la religion, en comprenant le contenu religieux à travers le mouvement même de la connaissance universelle. En d'autres termes, la dogmatique chrétienne croit que Dieu, par la mort du Christ, s'est radicalement séparé de l'humanité, qu'il est retourné à sa pure spiritualité abstraite du monde. Elle ne voit pas qu'avec cette mort, la représentation de l'"esprit divin immatériel » périt en retournant à soi enrichi d'un contenu qui lui faisait défaut au départ, que Dieu est maintenant véritablement "lui-même", c'est-àdire déterminé universellement et rempli de sens en ayant relevé son

36. Ph.G., p. 526; Ph.E., p. 687.

37. Ph.G., p. 512; Ph.E., p. 669.

38. Ph.G., p. 513 ; ibid. 
immédiateté abstraite par l'expérience de l'autre de lui-même. Les chrétiens fidèles à leurs dogmes ne voient pas non plus que chaque homme est luimême, non pas enfant, mais fils de Dieu, que l'humanité scelle la promesse du retour à soi de l'Absolu qui ne fait pas rayonner un paradis, un au-delà de notre monde, mais notre propre monde. Ne nous méprenons pas à ce sujet, l' "esprit se sachant comme esprit ${ }^{39}$ " (der sich als Geist wissende Geist) que chérit la philosophie hégélienne n'est pas celui qui s'oppose «extérieurement » à la nature, comme c'est le cas dans toute religion, mais celui qui s'y oppose "intérieurement» en étant associé au mouvement du connaître par lequel l'Absolu devient à soi-même un autre pour se regagner lui-même dans l'esprit de l'homme. En ce sens, l'on conviendra que l'"esprit qui se sait comme esprit", ou la pure spiritualité, que convoite notre auteur n'est donc pas celle qui préserve éternellement sa pureté en se détournant du sensible et du matériel qui pourraient la souiller, mais bien celle qui doit s'y maculer pour ensuite s'en purifier en devenant soi-même. Hegel nous dit en effet que "quel qu'il soit, chaque [contenu] a en lui la tache de la déterminité (den Makel der Bestimmtheit), [déterminité] dont est libre le pur savoir (das reine Wissen), qu'il peut mépriser tout comme il peut assumer n'importe laquelle ${ }^{40} »$. L'on comprendra que si le philosophe affirme que le "pur savoir", qui est celui de l'Esprit, peut mépriser le contenu déterminé, ce n'est qu'après l'avoir "assumé ", qu'après l'avoir lui-même expérimenté de fond en comble pour en «relever» (aufheben) l'immédiateté mensongère. La tentation d'entrevoir la pureté dont nous entretient Hegel comme ce qui est sans tache est toujours grande. On pourrait aisément y consentir, semble-t-il, en citant la prétention démesurée que le penseur accorde à sa Logique:

La logique $[\ldots]$ doit être saisie comme le système de la raison pure, comme le royaume de la pensée pure (das Reich des reinen Gedankens). Ce royaume est la vérité elle-même, telle qu'elle est sans voile en et pour soi; pour cette raison, on peut dire: ce contenu est la présentation de Dieu tel qu'il est dans son essence éternelle, avant la création de la nature et d'un esprit fini ${ }^{41}$.

Cette logicité, associée à la "vérité elle-même» (die Wahrheit selbst) et qui nous fait connaître Dieu "dans son essence éternelle avant la création de la nature et d'un esprit fini» (in seinem Wesen, vor der Erschaffung der Natur und eines endlichen Geistes ist), Hegel ne l'a pas simplement élaborée en logicien traditionnel, c'est-à-dire en n'utilisant qu'une pensée abstraite de tout contenu concret. Cette vérité — on l'a dit plus tôt - se présente dans une compréhension de la métaphysique religieuse où se dévoile l'Absolu. La Logique hégélienne assume l'Absolu comme le résultat

39. Ph.G., p. 531; Ph.E., p. 694.

40. Ph.G., p. 424; Ph.E., p. 560. Les interpolations sont de nous.

41. W.L. I., p. 21; S.L. I., p. 19. 
d'un long processus habitant, tout ce qui est, sans exception. Elle le fait en assumant un mouvement où intervient un Absolu animé, plus qu'on veut souvent le faire paraitre, par ces éléments sensibles et matériels qui font toujours signe, selon Hegel, vers le spirituel. Ainsi, nous dit le penseur:

De l'absolu il faut dire qu'il est essentiellement résultat, que c'est seulement au terme qu'il est ce qu'il est en vérité; et c'est en cela justement que consiste sa nature, d'être [quelque chose d']effectif, sujet, ou devenir-soi-même ${ }^{42}$.

Paradoxalement, ce «terme» par lequel l'Absolu a l'honneur d'être «quelque chose d'effectif», Hegel en fait aussi un «point de départ» en conceptualisant le vrai comme un "cercle qui présuppose son terme comme sa fin et $\left[l^{\prime}\right]$ a pour commencement ${ }^{43}{ }^{\prime}$. Cette conception circulaire de la vérité éclaire ce à quoi nous réfère ce Dieu «avant la création de la nature et d'un esprit fini » dévoilé par la Logique hégélienne. Ce que Hegel semble vouloir nous expliquer par cette lexie, c'est que le «royaume de la pure pensée " inclut l'entièreté de tout ce qui est en lui-même d'une façon non développée, ce qui veut donc dire que la "pensée pure» peut tout aussi bien se vérifier dans n'importe quel contenu matériel. La Phénoménologie de l'Esprit est soucieuse de nous faire comprendre qu'il est illusoire d'essayer d'atteindre l'Absolu dans la première forme concrète de sa manifestation, entendons dans la simple Nature qui semble séparée de l'esprit: "Là où nous désirons voir un chêne dans la force de son tronc et dans l'expansion de ses branches et les masses de son feuillage, dit-elle, nous ne sommes pas satisfaits quand au lieu de cela l'on nous montre un gland ${ }^{44}$.» Or, on a bien montré comment la nature matérielle se trouve présupposée logiquement au cœur de l'idée du "devenir soi-même de Dieu ", ou de la pensée pure, sur laquelle se penche la philosophie hégélienne. Ceci nous indique que Hegel pense l'origine du monde et sa finalité comme un tout. C'est l'exigence logique de l'universalité à "se lier à ce qui lui est autre", que Hegel a découverte au cœur même de l'idée de la connaissance en enseignant à Iéna, qui force toujours déjà la "pure pensée » à se manifester concrètement, à apparaître, non pas pour perdre sa pureté, mais bien, comme nous l'avons déjà expliqué, que pour mieux la préserver, ou du moins l'assumer. C'est ainsi, comme l'affirme Hegel dans son Encyclopédie, que «[le] logique devient la nature, et la nature l'esprit ${ }^{45}$ ». Entendons-nous bien ici, on ne passe pas $d u$ logique à la nature et de la nature à l'esprit dans le système hégélien, c'est le logique en tant qu'Absolu qui se fait luimême nature et cette dernière est Esprit. Cet éclaircissement peut paraître

42. Ph.G., p. 15 ; Ph.E., p. 83.

43. Ph.G., p. 14 ; Ph.E., p. 82.

44. Ph.G., p. $10 ;$ Ph.E., p. 76.

45. Hegel, System der Philosophie. Dritter Teil. Die Philosophie des Geistes, Sämtliche

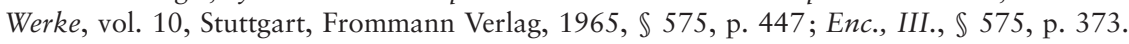


illusoire, mais ce n'est que lui qui peut nous faire comprendre que la seule extériorité dont on puisse parler lorsqu'on envisage l'Absolu d'un point de vue hégélien, c'est exclusivement celle qui concerne sa manifestation. En ce sens, Hegel nous parle, dans les dernières pages de sa Phénoménologie de l'Esprit consacrées au Savoir absolu, de la nature en termes d'" esprit extériorisé (der entäußerte Geist) [...] qui instaure le sujet (die das Subjeckt herstellt $)^{46} »$. Le philosophe allemand, on le voit avec cette dernière assertion, a effleuré l'un des principes de la science moderne, entendons l'idée d'une évolution de la réalité qui a conduit la nature à l'Esprit, l'animalité à l'humanité. Or, en ce qui concerne l'origine de l'espèce humaine, la philosophie hégélienne demeure contradictoire. D’un côté, Hegel semble nous indiquer que l'homme provient de l'animal, comme nous le laisse entendre la section Religion de sa Phénoménologie en affirmant que "la figure humaine se dépouille de la [figure] animale à laquelle elle était mêlée $e^{47}$; de l'autre, il certifie qu'en ce qui concerne l'homme et le monde, on peut soutenir qu' "un seul coup les crée tous les deux ${ }^{48}$ ».

Malgré les apparentes contradictions au niveau de l'origine de l'homme que l'on retrouve dans le discours philosophique de Hegel, on peut décidément conclure que l'Absolu hégélien s'aime d'un égal amour dans la pluralité de ses manifestations, même dégradantes, et qu'il peut franchement être abordé comme un amant «non narcissique » de lui-même. Nous avons en effet pu constater comment le mouvement logique mis à jour par la philosophie hégélienne arrive à supporter une telle affirmation. Une chose demeure certaine, c'est qu'après le passage de Hegel dans l'univers philosophique, aucune philosophie de l'esprit qui se respecte, même contemporaine, ne peut se permettre de considérer la nature comme quelque chose qui serait en dehors de son champ de juridiction. La nature est bien ce qui s'oppose sémantiquement à l'Esprit, et l'Esprit ce qui s'oppose à la nature. Mais ces deux termes ne font qu'un dans l'Absolu. Tout ce qui «est» est en fait lié à son autre par l'acte de connaître. C'est là, certainement, l'un des plus précieux enseignements que nous devons à la pensée hégélienne, qui ne maltraite la nature que lorsque cette dernière n'est pas saisie comme en relation avec l'Esprit absolu représenté dans la religion chrétienne, ou, pour employer des mots sûrement chers à Hegel, en raison de sa compréhension de la religion, lorsque das Mysterium des Brods und Weins ist noch nicht Mysterium des Fleisches und Blutes ${ }^{49} .$.

46. Ph.G., p. 530 ; Ph.E., p. 693.

47. Ph.G., p. 462 ; Ph.E., p. 607.

48. Hegel, Jenaer [illisible], Philosophische Bibliotek, vol. 333, Hambourg, Felix Meiner; La Philosophie de l'esprit (1805), trad. de G. Planty-Bonjour, Paris, Presses Universitaires de France, 1982, p. 120.

49. «[...] le mystère du pain et $[\mathrm{du}]$ vin n'est pas encore mystère de la chair et [du] sang. »Ph.G., p. 472; Ph.E., p. 619. 